Anuario Latinoamericano

Ciencias Políticas

y Relaciones Internacionales

vol. 3, 2016

pp. 169-182

\section{La construcción de un nuevo modelo de seguridad en América del Sur: una perspectiva desde Brasil}

\section{The construction of a new security model in South America: a perspective from Brazil}

\author{
Alexandre Fuccille \\ UNIVERSIDADE ESTADUAL PAULISTA (UNESP) \\ BRASIL \\ fuccille@hotmail.com
}

\title{
RESUMEN
}

En América del Sur, en este siglo, hemos presenciado una transición de un ajuste de seguridad colectiva hacia un modelo de seguridad cooperativa. Brasil ha sido protagonista en ese proceso. En este sentido, el presente artículo pretende realizar una evaluación del estado del arte de este debate, problematizando la participación brasileña en la creación del Consejo de Defensa Suramericano y, al mismo tiempo, enfocando las antinomias que han caracterizado ese proceso, la postura de Brasilia en lo que se anunciaba un nuevo modelo de defensa subregional y las disputas interregionales como un componente que hace muy difícil el planteamiento de nuevas culturas institucionales.

PALABRAS CLAVE: Consejo de Defensa Suramericano, cooperación, seguridad y defensa, política externa brasileña, Complejo Regional de Seguridad.

\begin{abstract}
In South America this century we've witnessed a transition from a collective security arrangement towards a cooperative security model. Brazil has been a central actor in this process. In this sense, the present article intends to evaluate the current stage of this debate, discussing the Brazilian participation in the creation of the South American Defense Council and, at the same time, approaching the antinomies that have been characterizing this process, Brazil's central government behavior in what was being announced as a new model of sub-regional defense, as well as the inter-regional disputes as an ingredient that greatly impedes the appearance of new institutional cultures.
\end{abstract}

KEYWORDS: South American Defense Council, cooperation, security and defense, Brazilian foreign policy, Regional Security Complex. 
En el período de la post Guerra Fría, América del Sur ha intentado hacer nuevos ajustes en las más variadas áreas de seguridad y defensa. En una región tradicionalmente marcada por una baja cooperación institucional en los más diferentes campos, llama la atención la rapidez con que se aspira a instituir nuevos cánones en lo referente a la temática descrita.

Fundamentalmente, se percibe un esfuerzo que pretende representar el cambio de una medida anticuada de seguridad colectiva hacia un modelo más dinámico de seguridad cooperativa, sin embargo de relieves aún inciertos y cuyo resultado final sigue en proceso, privilegiando sobre todo la voluntad política y liderazgo de los gobernantes por encima de la institucionalidad.

En este sentido, un conjunto de cuestiones permanece todavía en interrogantes: ¿qué hemos presenciado en América del Sur, una corrida armamentista o reequipamiento? ¿y los históricos contenciosos fronterizos, han sido superados? ¿los espacios e identidades nacionales son respetados recíprocamente? ¿cómo se pueden evitar los desequilibrios y las asimetrías donde la economía/ PIB - así como los gastos en el área de defensa - sólo en Brasil representan más de la mitad de todos los otros países del subcontinente sumados? ¿se puede discurrir - definitivamente - referente a la superación de la dicotomía Pacífico (arco de inestabilidad/CAN) versus Atlántico (arco de estabilidad/Mercosur)? ¿cómo la cuestión amazónica se presenta en la actualidad? ¿el riesgo de balcanización de países de la región es cosa del pasado? ¿la "Zona de Paz y Cooperación del Atlántico Sur" es una realidad efectiva? ¿la tutela estratégica norteamericana ha sido refrenada? ¿el Consejo de Defensa Suramericano/ CDS ha correspondido a las expectativas que en él se depositan? ¿los días están contados para los ajustes basados en el principio de la seguridad colectiva para la región? Por último, éstas y otras interrogantes suscitan un prometedor debate que debe ser enfrentado.

Con apoyo del análisis cualitativo de los documentos oficiales producidos en el ámbito del Consejo de Defensa Suramericano y de los Ministerios de Defensa y de las Relaciones Exteriores brasileños, llevándose en cuenta el contexto internacional y nacional de los demás 11 países participantes del CDS, se va a resaltar una mirada cronológica de los hechos, aunque, a principio, el método de investigación no esté en conformidad con el orden de esos mismos hechos, pues el privilegio es la búsqueda de las variables predeterminadas consonante a la metodología definida para la investigación. Por otra parte, como eje central de nuestra reflexión, sigue como base de argumentación la teoría de los Complejos Regionales de Seguridad - de acuerdo con el propuesto por

${ }^{1}$ Este trabajo fue parcialmente financiado por la Coordenação de Aperfeiçoamento de Pessoal de Nível Superior (CAPES) y por el Ministério da Defensa do Brasil como parte del proyecto de investigación $A$ inserção brasileira em um sistema internacional em transformação: alcances e possibilidades na Segurança Internacional e na Defesa. 
Buzan y Wæver - y de otras ideas centradas en la "Escuela de Copenhague", para que se pueda mejor comprender la cuestión de la seguridad en América del Sur desde una perspectiva más amplia.

\section{EI Consejo de Defensa Suramericano/CDS como un punto de inflexión}

El Consejo de Defensa Suramericano (CDS), creado a fines de 2008, se presenta como una innovación histórica en una región donde, si bien podemos afirmar que en el pasado se caracterizó por importantes conflictos y disputas por liderazgo en el plan subregional, a pesar de haber desarrollado considerables adelantos en las últimas décadas, no es posible afirmar que estamos en otro opuesto de la cooperación, que sería la integración con marcados elementos de supranacionalidad. En ese medio de camino, el CDS parece destacarse en mayor medida como una instancia de cooperación, donde la coordinación de políticas en temas relativos a su área parece ser el conductor de la asociación de los países suramericanos.

Visto como un verdadero punto de inflexión referente a la clásica idea de seguridad colectiva heredada de la Guerra Fría - bajo el liderazgo norte-americano -, el CDS aparece como una admirable iniciativa en una región tradicionalmente marcada por una baja autonomía y acumulo en materias relacionadas a la temática de defensa. Sin embargo, desde su creación hasta su pleno funcionamiento, la construcción y consolidación de este ajuste institucional ubicado en el interior de la Unión de Naciones Suramericanas (UNASUR) no ha ocurrido ordenadamente, con descompás, contradicciones, zonas escusas de actuación y déficits de conducción democrática en muchos de los temas que se propone a tratar.

El final de la Guerra Fría, la intensificación del proceso de globalización y lo que se acordó nombrar como «nuevas amenazas» (normalmente vía actores noestatales) evidenció la crisis de un modelo de seguridad hemisférico (o panamericano) que presentaba problemas desde hace mucho tiempo. La creciente erosión de la legitimidad del modelo de seguridad colectiva del período anterior es confirmada en, por ejemplo, la poca efectividad del Tratado Interamericano de Asistencia Recíproca (TIAR), la Junta Interamericana de Defensa (JID) y hasta la propia Organización de los Estados Americanos (OEA). Lo anterior, combinado con un relativo descenso estratégico de los EE.UU. en la región, así como nuevas prioridades por parte del Departamento de Estado estadounidense pos 11 de septiembre de 2001, acabaron por consolidar un imaginario en América del Sur de espacio geográfico posible y pasible de desarrollar políticas más independientes, advenidas de una mayor margen de autonomía.

En el presente siglo se ha instaurado una situación completamente distinta de la que tuvimos por décadas, producto de la emergencia de gobiernos nacionales progresistas y/o a la izquierda en la región - aunque de diferentes matices y carac-
La construcción de un nuevo modelo de seguridad en América del Sur: una perspectiva desde Brasil

Alexandre Fuccille 
terizados por una heterogeneidad que no se puede desconsiderar -, importantes alteraciones en la agenda internacional pos septiembre de 2001, crecientes reveses en los más diversos campos y una declinación relativa norteamericana en el sistema internacional, así como por la salida del estado de hibernación en que quedó el oso ruso a lo largo de 1990 y el espectacular crecimiento chino.

Ya a mediados del año 2000, en la I Cumbre Suramericana realizada en agosto/septiembre en Brasilia, por iniciativa, en aquel entonces, del Presidente Fernando Henrique Cardoso, empezó a delinearse la necesidad de una mayor intensificación de las relaciones de los países de la región. Si geografía es destino, como se dice en geopolítica, entonces Brasil y sus vecinos parecían estar despertando de un largo período de letargia e inacción, conscientes de la necesidad de acuerdo para una agenda común de oportunidades y desafíos. De este modo, en el naciente siglo XXI las reuniones se vuelven más rutinarias y nuevos ajustes institucionales son pensados e implementados.

A la vez, la toma de posesión del Presidente Luiz Inácio Lula da Silva, en enero de 2003, traería importantes cambios no sólo internos, sino que también externos, producto de la dimensión adquirida como de un mayor player regional y con base en sus propias dimensiones físicas, políticas, económicas y militares. ${ }^{2}$ En el área de defensa, el hecho de que Brasil ha presentado una gran variedad de nuevos documentos legales de alto nivel como la Política de Defensa Nacional (PDN) de 2005, la Estrategia Nacional de Defensa (END) de 2008 y, por fin, el Libro Blanco de Defensa Nacional (LBDN) de 2012, cada vez más evidenciaban la priorización del espacio suramericano. ${ }^{3}$

Particularmente, la institución del CDS, pensado y entendido como conditio sine qua non a la construcción de una vigorosa comunidad de seguridad o, aun, para la transformación del Complejo Regional de Seguridad como proponen Buzan y Wæver (2003: 27-39) $)^{4}$ - hacia un modelo centrado, empezaron a conducir muchas de las reflexiones y estudios que abarcan esta temática. ${ }^{5}$ En primera instancia, conviene destacar que, entre la presenta-

2 Corroborando estos elementos, el recuerdo de que muchas naciones suramericanas son más pequeñas geográficamente que muchas entidades federativas, el estado brasileñas (e.g., si el Estado de Amazonas fuera un país sería el $3^{\circ}$ más grande de América del Sur - seguido de Brasil y Argentina), menos poblados (e.g., el estado de Minas Gerais cuenta con una población mayor que Bolivia, Paraguay y Uruguay sumados), de menor peso económico (e.g., si el estado de São Paulo fuera un país sería la $2^{\circ}$ mayor economía del subcontinente, perdiendo solamente para el propio Brasil), entre otros puntos, no pueden evitar que se provoque un malestar entre sus vecinos.

3 Se puede consultar esta literatura en línea por el acceso de http://www.defesa.gov.br/estado-e-defesa-editoria/artigo-de-teste/estado-e-defesa, fecha de consulta: 28 septiembre 2015.

4 Para éstos la estructura de seguridad internacional es formada por la superpotencia Estados Unidos de América, cuatro grandes potencias (Unión Europea, China, Japón y Rusia) a este ajuste se ha denominado de $1+4-$, más las potencias regionales (que se pueden presentar en el interior del CRS conformado en términos unipolar, bipolar o multipolar).

5 Más discusión acerca de los CRSs y sobre América del Sur se puede encontrar en Fuccille y Rezende 2013, en línea: http://www.scielo.br/pdf/cint/v35n1/a03v35n1.pdf, fecha de consulta: 30 junio 2015. 
ción de la propuesta que se inició con diálogos bilaterales entre bastidores a lo largo de 2006-2007, ${ }^{6}$ la exposición pública y posterior aprobación del CDS en la Reunión Extraordinaria del Consejo de Jefas y Jefes de Estado y de Gobierno de UNASUR en diciembre 2008, diversas fueron las articulaciones para superar las diferencias de forma y contenido entre sus 12 miembros, así como sepultar la idea de que tal instrumento pretendía consolidar la hegemonía brasileña en la región. Incluso, desde su inicio, el CDS ha sido pensado e idealizado como locus de concertación e interlocución entre sus integrantes, capaz de fomentar el intercambio en los campos de seguridad y defensa, cuyas determinaciones sólo son válidas, si son acordadas por consenso y con previsión de al menos un encuentro anual ordinario entre sus ministros de Defensa.

Sin embargo, cuando se analiza la cronología de los temas que componían la agenda prioritaria (y también la no-prioritaria) que sería discutida en este esfuerzo integrador suramericano, no existía ninguna mención a la temática de seguridad y defensa, solo de forma marginal. Cabe preguntarse entonces, ¿qué habrá cambiado para que el tema haya adquirido tal protagonismo?

Para algunos participantes de este proceso, dentro de los fundamentos para acelerar la conformación de este ajuste - por lo menos desde el punto de vista brasileño - se encontraban los siguientes:

(1) La posibilidad de desborde de las llamadas „nuevas amenazas” a otros países, como el narcotráfico colombiano para Brasil y la contienda entre Colombia, Ecuador y Venezuela por la muerte de Raúl Reyes.

(2) Los conflictos subregionales, como los contenciosos que involucran a Perú y Ecuador, Bolivia y Chile, entre otros.

(3) Los movimientos autóctonos difundiendo un sentimiento separatista, como ilustran Bolivia y Paraguay.

(4) Frenar los ímpetos bolivarianos en la región, sobre todo vía aislamiento de Venezuela y/o de los países integrantes de la Alianza Bolivariana para los Pueblos de Nuestra América (ALBA).

(5) Evitar una carrera armamentista en la región, con la adopción creciente de medidas de confianza mutua (CBMs).

(6) Solidificar el Atlántico Sur como un área de paz, libre de armas nucleares y de vital proyección de poder al Brasil, no permitiendo la presencia de potencias extra-regionales (como el caso del Reino Unido y la cuestión Malvinas/Falklands).

(7) Impedir la materialización de una política estratégica de seguridad hemisférica definida a partir de los Estados Unidos para el subcontinente.

6 Para una cronología de creación del CDS y disputas envolviendo su institución, ver Medeiros Filho 2010, en línea: http://www.brasa.org/_sitemason/files/fLdvB6/Oscar\%20Medeiros\%20 Filho.pdf, fecha de consulta: 13 julio 2015; Carvalho 2009; y Teixeira 2011. Para un balance mayor, cf. Pagliari 2010.
La construcción de un nuevo modelo de seguridad en América del Sur: una perspectiva desde Brasil

Alexandre Fuccille 
(8) Por último, pero no menos importante, concretizar los objetivos estratégicos de la Política Externa Brasileña de consolidación de un proceso de integración suramericano. ${ }^{7}$

Efectivamente, más que nada, para que el desarrollo brasileño pudiera acelerarse y consolidarse su deseo de player global, la estabilidad jugaba un papel fundamental no sólo para el crecimiento pleno de sus potencialidades, sino que también para mostrar al mundo su desempeño como proveedor de una importante estabilidad regional.

Es importante dejar en claro que, más allá de los éxtasis triunfalistas que a las veces adquiere la Política Externa Brasileña en el período reciente, solamente fue posible erigir una estructura como el CDS por la opción de no confrontar abiertamente con Washington. Consciente de sus demasiadas limitaciones en el campo de las capabilities, Brasilia optó por una especie de low profile en la construcción de su liderazgo (que no se percibe tampoco se recibe como natural en la región, al contrario de lo que muchos defienden) referente a las temáticas aquí problematizadas. Indirectamente, sin la intención de sugerir cualquier relación mecánica centro-periferia/Washington-Bogotá, los Estados Unidos supieron valerse de los estrechos vínculos político-ideológicos con el país andino y la colaboración en el área castrense vía el Plan Colombia, para asegurar lo que no pretendían para la región.

Amparándose en el recelo de una Colombia aislada, rodeada de gobiernos izquierdistas, el Presidente George W. Bush supo utilizarse de la ascendencia y otros vínculos con el Presidente Álvaro Uribe para la creación de una estructura suavizada (no-operativa), como es el CDS. ${ }^{8}$ En la práctica, la no participación de Colombia - como se llegó a considerar como hipótesis - atentaría contra la esencia y el sentido del Consejo de Defensa Suramericano. Ese escenario sería problemático para la tentativa brasileña de liderar una armonización de las percepciones de securitización de los Estados de la región de los dos Subcomplejos Regionales de la América del Sur (Norte Andino y del Cono Sur).

Con la demanda colombiana contemplada, se llegó a un texto final donde, en lo que se refiere a la naturaleza del CDS, se propugnaba su creación como una "instancia de consulta, cooperación y coordinación en materia de Defensa" en los marcos de la institucionalidad y principios de la UNASUR. Conforme su documento constitutivo, sus objetivos generales son: " (a) consolidar Suramérica como una zona de paz, base para la estabilidad democrática y el desarrollo integral de nuestros pueblos, y como contribución a la paz mundial; (b) construir una identidad suramericana en materia de defensa, que tome en

7 Notas personales a partir de charlas off the record con miembros del Itamaraty y Ministerio de Defensa brasileño.

8 En otro extremo, tuvimos una Venezuela histriónica que defendía la creación de un instrumento militar operacional común - o sea, una alianza militar - conocida como Organización del Tratado del Atlántico Sur (OTAS, siguiendo el mismo modelo de la Organización del Tratado del Atlántico Norte/OTAN). En la gran media, esta propuesta fue acuñada "OTAN del Sur" o aun "OTAN de los pobres". 
cuenta las características subregionales y nacionales y que contribuya al fortalecimiento de la unidad de América Latina y el Caribe; (c) generar consensos para fortalecer la cooperación regional en materia de defensa". Más allá de estos objetivos generales, fueron definidos once objetivos específicos. ${ }^{9}$

\section{Balance de la actuación del CDS: 2009-2014}

Resumidamente y pensando en términos de región y sus desdoblamientos en el Complejo de Seguridad, se puede mencionar la creación de mecanismos como el "Tratado Americano de Soluciones Pacíficas"/Pacto de Bogotá (1948), el "Tratado para la Proscripción de las Armas Nucleares en la América Latina y el Caribe"/Tratado de Tlatelolco (1967), la "Zona de Paz y Cooperación del Atlántico Sur"/ZPCAS (1986), el "Acuerdo de Mendoza" entre Argentina, Brasil y Chile (1991) que prohíbe la proliferación, la posesión y el uso de armas químicas y biológicas, la "Zona de Paz Suramericana"/Comunicado de Brasilia (2000). Asimismo se puede agregar la inauguración de las "Reuniones de Ministros de Defensa de América del Sur" (la primera ocurriendo en Rio de Janeiro en 2003), además de la intensificación de las relaciones bilaterales entre Brasil y Argentina desde la década de 1980 en el área nuclear con la abdicación de la fabricación y empleo por ambos de armas atómicas y la institución de la Agencia Brasileño-Argentina de Contabilidad y Control de Materiales Nucleares/ABACC (único organismo binacional de salvaguardias nucleares del mundo), culminando en el «Acuerdo Bilateral sobre Cooperación en Materia de Defensa»/DCA firmado en 2007. ${ }^{10}$

9 Según el Artículo 5 del Estatuto del CDS, son ellos: (a) avanzar gradualmente en el análisis y discusión de los elementos comunes de una visión conjunta en materia de defensa; (b) promover el intercambio de información y análisis sobre la situación regional e internacional, con el propósito de identificar los factores de riesgo y amenaza que puedan afectar la paz regional y mundial; (c) contribuir a la articulación de posiciones conjuntas de la región en foros multilaterales sobre defensa, dentro del marco del artículo $14^{\circ}$ del Tratado Constitutivo de UNASUR; (d) avanzar en la construcción de una visión compartida respecto de las tareas de defensa y promover el diálogo y la cooperación preferente con otros países de América Latina y el Caribe; (e) fortalecer la adopción de medidas de fomento de la confianza y difundir las lecciones aprendidas; (f) promover el intercambio y la cooperación en el ámbito de la industria de defensa; (g) fomentar el intercambio en materia de formación y capacitación militar, facilitar procesos de entrenamiento entre las Fuerzas Armadas y promover la cooperación académica de los centros de estudio de defensa; (h) compartir experiencias y apoyar acciones humanitarias tales como desminado, prevención, mitigación y asistencia a las víctimas de los desastres naturales; (i) compartir experiencias en operaciones de mantenimiento de la paz de Naciones Unidas.; (j) ntercambiar experiencias sobre los procesos de modernización de los Ministerios de Defensa y de las Fuerzas Armadas; (k) promover la incorporación de la perspectiva de género en el ámbito de la defensa. Para más esclarecimientos sobre los principios, estructura y funcionamiento que orientan el CDS, ver http://www.ceedcds.org.ar/ Espanol/09-Downloads/ESTATUTO_CDS.pdf, fecha de consulta: 28 agosto 2015.

${ }^{10}$ A esos podrían sumarse las iniciativas brasileñas (todas de este siglo) de los Acuerdos Marco Bilaterales con Bolivia, Chile, Colombia, Ecuador, Paraguay, Perú y Uruguay, además de
La construcción de un nuevo modelo de seguridad en América del Sur: una perspectiva desde Brasil

Alexandre Fuccille 
Por último, no se debe ignorar la tradición de intentar distender el ambiente de seguridad y defensa regional, ${ }^{11}$ además de servir para el propósito de aproximar los Subcomplejos Regionales de Seguridad Norte-Andino y del Cono Sur, destacando el papel central brasileño en ese proceso.

Más allá del gran interés brasileño, autor de la iniciativa del Consejo de Defensa Suramericano, idiosincrasias locales, regionales y extra-regionales parecen haber fortalecido el esfuerzo para la concretización de esta iniciativa. En un breve periplo por el subcontinente se percibe que el CDS podría venir a cumplir diferentes designios, en donde todos eventualmente podrían ganar algo. Desde la perspectiva argentina, era de interés aglutinar nuevos actores a la disputa de que "las Malvinas son argentinas" y todos los desdoblamientos que esta cuestión encierra; pasando por un Paraguay acosado por problemas internos que culminó en el surgimiento del grupo guerrillero Ejército del Pueblo Paraguayo (EPP); una Venezuela que, a pesar de abajo del modelo deseado por el comandante Chávez (una alianza militar), lo veía como un importante instrumento para que evitara ser encapsulado o incluso sufrir una acción directa por parte de los EE.UU.; a los otros bolivarianos de la región, Bolivia y Ecuador, a quienes les interesaba buscar garantías ante acciones desestabilizadoras a ejemplo de lo que se pasó con y después del episodio de Angostura; para Colombia, aunque reticente a principio y temiendo al aislamiento, vio la posibilidad de un mayor compromiso y comprensión de los países vecinos con el flagelo de su conflicto interno; Perú y Chile, con preparos y capacidades militares bastante distintos, buscaban reforzar la solución de los litigios por vías diplomáticas y eventualmente por medio de tribunales internacionales; al norte, Guyana y Surinam veían una posibilidad para la intensificación de su suramericanización (en contraste con la histórica vocación caribeña); y, finalmente, con el CDS, Uruguay conseguía superar sus restricciones a una preocupante colaboración militar entre Brasil y Argentina que causaba gran incomodidad en Montevideo.

De todas formas, por diferentes lecturas y comprensiones, parecía que el CDS no sería el tipo de estructura que se opondría o dificultaría el ejercicio de las soberanías nacionales en América del Sur y los países concordaban en cuanto a la oportunidad y la conveniencia. Seguramente, el camino estaba libre para las oportunidades de cambio de un modelo caduco de seguridad colectiva a una nueva forma más dinámica de seguridad cooperativa. ¿Pero, será el Consejo de Defensa Suramericano un mecanismo multilateral estable, útil y eficaz en situaciones conflictivas?

los Acuerdos Marco de seguridad regional firmados por el Mercosur con Bolivia, Chile, Colombia, Ecuador, Perú y Venezuela.

${ }^{11}$ Unos ven en estas iniciativas, que auxiliaron en la ausencia de guerras en el último siglo, el surgimiento de una comunidad pluralista de seguridad en la región. En específico, cf. Hurrel 1998 y Kacowicz 2005. En oposición a esta lectura optimista, Mares propugna la existencia de una "paz violenta", caracterizada, entre 1884 y 1993, por 127 disputas interestatales militarizadas (DIMs) en la esfera suramericana, algunas de ellas ya en parejas democracia/democracia. Mares 2001: 39-43. 
En sus años iniciales, el CDS ha aparecido como un importante espacio de concertación donde, frente a las idiosincrasias regionales/ideológicas entre la Comunidad Andina de Naciones (CAN), el Mercado Común del Sur (Mercosur), o aun la Alianza Bolivariana para las Américas (ALBA), importantes avances han sido registrados, como la definición de Planes de Acción comunes en la temática de seguridad y defensa, el establecimiento de medidas de confianza mutua, la creación del Centro de Estudios Estratégicos de Defensa (CEED) en la ciudad de Buenos Aires, la institución de la Escuela Suramericana de Defensa (ESUDE) para altos estudios en Quito, la construcción de una metodología común de medición de los gastos en defensa, un importante intercambio en materia de formación y capacitación militar, entre otros puntos igualmente meritorios. Sin embargo, aún permanecen algunos desafíos.

Una vez concretada la fase embrionaria de estructuración y concertación de contenidos mínimos, la gran misión es continuar con los avances basados en una metodología que impone, obligatoriamente, la necesidad de concordancia unánime entre sus miembros - o, al menos, no objeción explícita por cualquier uno de los integrantes. La regionalización de la estructura de seguridad y defensa en el subcontinente prosigue, sin embargo, con nuevos desafíos.

Pero vamos a ver: de modo muy breve y de cierta forma como desafíos a la implementación del mandato del CDS a partir de 2009, se ha tenido "una propuesta peruana, recomendando desarmamiento, desmilitarización, un pacto de no agresión y la creación de una Fuerza de Interposición de Paz; una propuesta chilena, recomendando una Fuerza Combinada de Defensa Suramericana; y una propuesta ecuatoriana, propugnando un código de conducta y mecanismos de reducción de gastos militares" (Abdul-Hak 2013: 179 y sig.). Por último, una considerable heterogeneidad de puntos de vista para la región considerada como la más desmilitarizada del mundo, que evidencia las dificultades para la consolidación del CDS, su intensificación (en virtud de la actual baja institucionalización), pone en duda su eficacia y efectividad, así como explicita la polisemia que marca los conceptos de seguridad y defensa en la región, además de las disparidades y asimetrías en los campos social, económico y militar que caracterizan los países miembros.

De hecho, el CDS aún no ha pasado por ninguna prueba de fuego capaz de poner en jaque la eficacia y eficiencia de esta estructura institucional. A la vez, la conocida como agenda negativa que caracterizó la relación EE.UU. - América del Sur en el período post Guerra Fría, centrada en el combate al tráfico de drogas, terrorismo y otros delitos de naturaleza policial, fue parcialmente superada. ${ }^{12}$ A despecho de eso, los Subcomplejos Regionales de Seguridad del Cono Sur y Norte-Andino vivencian dinámicas aún bastante dispares entre

${ }^{12}$ La creación de instancias como el Consejo Suramericano sobre el Problema Mundial de las Drogas, apartado del Consejo de Defensa Suramericano, ilustra como una mayor madurez más allá de la habitual cruzada prohibicionista norte-americana de militarización en el combate al narcotráfico - en diferentes temas se ha desarrollado en el subcontinente.
La construcción de un nuevo modelo de seguridad en América del Sur: una perspectiva desde Brasil

Alexandre Fuccille 
sí, que carecen de mayor articulación y organicidad. En este sentido, para que se pueda ejemplificar, la aprobación en 2014 por parte de la mayoría en el Congreso colombiano (con casi $90 \%$ de los votos) del acuerdo de cooperación entre Colombia y OTAN, indica señales contradictorios sobre el deseo de una mayor profundización de la cooperación en defensa en el subcontinente. ${ }^{13}$

El comportamiento brasileño, principal agente de creación del Consejo de Defensa Suramericano, por su vez, carece de coherencia y se presenta innumerables veces de forma diacrónica. Si por un lado, en el más alto nivel político, Brasilia trabaja por la materialización de este ajuste institucional (que ya representa 2/3 del destino de las exportaciones brasileñas en armamentos), ${ }^{14}$ incluso en una especie de liderazgo consentido por parte de Washington y sin confrontarla en el nivel global, por otro, se descuida de comprometer el Ministerio de Defensa y, más particularmente, los militares brasileños, a actuar de forma más decisiva y decidida por la concretización de esta importante iniciativa y de sus mecanismos operativos.

\section{Perspectivas}

El intento de dar vigor a las nuevas estructuras en paralelo a la pérdida de fuerza del sistema interamericano demuestra que las perspectivas entregadas pueden ser prometedoras. En ausencia de una comunidad de seguridad en América del Sur y más allá del permanente dilema de seguridad - conforme a lo propuesto por Herz hace más de medio siglo - que sigue siendo válido, la armonización de esfuerzos en la temática de defensa y una mayor capacidad disuasoria regional podría ser de interés de todos los países del subcontinente, desalentando aventuras y/o acciones hostiles de manera aislada o colectiva.

Apropiadamente, desde nuestro punto de vista, temas complejos y que no deben ser afectos a la defensa nacional como el empleo de los militares en seguridad pública, combate al narcotráfico y otras tareas de policía se ha dejado

${ }^{13}$ En junio de 2015 la Corte Constitucional colombiana declaró inexequible el acuerdo entre Colombia y la Organización del Tratado del Atlántico Norte (OTAN) sobre cooperación y seguridad de información (o Ley no 1734). Cf. «Corte tumba tratado con OTAN sobre seguridad», http://www.eltiempo.com/politica/justicia/corte-tratado-con-otan-sobre-seguridad-de-informacion/15884939, fecha de consulta: 12 septiembre 2015.

${ }^{14}$ Es posible afirmar que, desde la primera reunión en la historia de ministros de Defensa de América del Sur ocurrida en 2003 en Rio de Janeiro en el interior de la LAAD - mayor feria del mercado de defensa de América Latina -, aún en el inicio del gobierno de Lula da Silva, la idea de una base industrial de defensa compartida y a fin de garantizar escala ya informaba en larga medida los movimientos brasileños en este área. La estrategia de aumentar ganancias extendiendo las relaciones económico-comerciales con los vecinos suramericanos sigue en curso, garantizando los mejores resultados tanto cuantitativamente cuanto cualitativamente a la balanza de comercio brasileña - aquí, más de $3 / 4$ de las exportaciones fueron de productos manufacturados, contrastando con el desempeño de este país para mercados extra-regionales. Ver datos en http:// www.mdic.gov.br/sitio/interna/index.php?area=4, fecha de consulta: 17 mayo 2015. 
de lado de las atribuciones del Consejo de Defensa Suramericano, a pesar de que éste no explicite defensa contra quién o qué. Sin embargo, aunque una condición necesaria, esto no es suficiente.

Como debidamente es resaltado por Saint-Pierre y Palacios Junior:

El hiato entre las declaraciones politicas y las acciones de cooperación en Defensa es agravado por el hecho de que no han sido elaboradas y asimiladas nuevas doctrinas para la cooperación suramericana. En líneas generales, los relieves estratégicos aún reflejan anacrónicamente las expectativas anteriores al fin de la Guerra Fría. (Saint-Pierre y Palacios Junior 2014: 35)

Además, en varias circunstancias, Brasil no ha tenido éxito, por decir lo menos, en erigir un discurso coherente y que parezca creíble a los demás socios regionales, teniendo como consecuencia un perfil de identidad aún no claramente delineado. Al discurrir sobre el subcontinente y la relación entre éste y Brasil, mientras el Presidente Cardoso destacaba que "sólo tendremos éxito si somos capaces de ser compañeros de los otros países. No podemos oprimir, aprovecharnos innecesariamente. La ventaja es estratégica, no es para mañana”. En tanto, algunas veces hay afirmaciones como la del Presidente Lula donde se destaca que "es impresionante como todos esos países están casi a exigir que Brasil asuma el liderazgo en América del Sur". ${ }^{15}$

A pesar de los esfuerzos hechos por Lula da Silva para que la integración regional pudiera ser puesta en práctica y la acción que el Banco Nacional de Desarrollo Económico y Social (BNDES) ha jugado para concretizar la Iniciativa para la Integración de la Infraestructura Regional Suramericana (IIRSA), como uno de los brazos de la política externa brasileña, en gran parte existe un sentimiento general que observa a Brasil como una potencia hegemónica, gigante e imperialista, impregnando los espíritus y pensamiento de gran parte de los vecinos suramericanos. ${ }^{16}$ Para que tal percepción se desvanezca, es necesario avanzar, reduciendo efectivamente las importantes asimetrías que aún caracterizan a los países de la región y apostar por la integración regional de manera seria (no sólo como mecanismo de salvaguardia a amenazas externas comunes de diferentes tipos y/o un tipo de soft balancing). No solamente los vecinos menores, sino también Brasil sería muy favorecido si tal proceso ocurriera. Actualmente, la dificultad de la Presidenta Dilma Rousseff consiste en un resiliente marco internacional de crisis, que impacta directamente los

15 «Para América do Sul, liderança brasileira ainda é promessa». En línea: http://www.bbc. co.uk/portuguese/reporterbbc/story/2008/03/080303_ams_abre1_diplomacia.shtml, fecha de consulta: 12 agosto 2015 .

${ }^{16}$ Para que se hable sobre Brasil en el contexto suramericano, necesariamente implica discurrir sobre números grandiosos: $48 \%$ del área total del subcontinente, 50\% de su población, 59\% de su economía/PIB (deteniendo aproximadamente $75 \%$ de la base industrial y $2 / 3$ de su agricultura), $42 \%$ de la producción de petróleo, responsable por casi 3/4 de los desembolsos centrados en la infraestructura de la integración regional, entre otros indicadores igualmente superlativos.
La construcción de un nuevo modelo de seguridad en América del Sur: una perspectiva desde Brasil

Alexandre Fuccille 
planes doméstico, regional y global - limitando la capacidad de acción del Estado brasileño en el sistema internacional -, así como la adopción de una política externa mucho más tímida y menos pro-integración regional que la de su antecesor y padrino político Lula da Silva.

Empiezan a ocurrir ciertos cambios y, solamente para ilustrar, podríamos citar la IIRSA (reeditada en 2009 bajo la forma de Consejo Suramericano de Infraestructura y Planeamiento/COSIPLAN, protegido en el interior de la UNASUR). Las zonas privilegiadas siguen siendo las mismas (transportes, energía y comunicaciones), pero con un enfoque distinto. Mientras en la IIRSA la lógica del business y de los corredores de exportación informaban de forma más amplia aquella iniciativa, en el COSIPLAN el imperativo de la integración regional (aunque sin abandonar una lógica del business), de fomento e incremento de comercios intra-bloques y de una nueva estructura económica/ desarrollo es lo que se destaca. Sin duda, el "regionalismo post hegemónico" posibilitó que se piense otro tipo de integración.

Sin embargo, la construcción de una activa e altiva comunidad de seguridad con vistas a la estabilidad regional aparece como un tema y tarea central en los marcos más amplios de ese proceso, una vez que aun con la decisiva participación brasileña en dirimir desconfianzas, crear entendimientos y facilitar el diálogo, la tan anunciada identidad suramericana en materias de defensa hasta este momento es una promesa no realizada. Pero, las políticas integracionistas avanzan en diferentes velocidades, a veces de forma más acelerada en el campo político - véase el Consejo de Defensa Suramericano -, seguido por un descompás en el campo económico y otros, así como de una estructura institucional más sólida capaz de dar soporte a una integración más profunda y proficua. No obstante, nos aventuramos a decir que nunca, aún con Simón Bolívar, las posibilidades de cooperación han sido tan grandes como en la actual escena histórica.

\section{Consideraciones finales}

Si ciertamente la integración regional ha funcionado como una estrategia para garantizar más voz (y fuerza) a los pleitos de la región en un mundo de crecientes asimetrías de poder, esto no puede ofuscar la absoluta fragilidad que tal instrumentalización de este importante proceso representa para una cooperación más sistémica, a pesar de avances específicos. El cambio de la seguridad colectiva a la seguridad cooperativa es un hecho, pero, si Brasil efectivamente quiere liderar, es necesario deseo y movilización para tanto y que esté consciente de que esto tiene un precio (¡el que no es barato!).

Brasil repetidamente se ha presentado de manera ambivalente en los marcos más amplios de ese proceso, liderándolo y pretendiendo presentarse en el papel de paymaster (que no se limita solamente al aspecto económico), por veces retrayéndose y subordinando esta opción que, desde nuestro punto de vista, más allá que una oportunidad es una necesidad. Sin embargo, es esen- 
cial reconocer que, sea la sociedad brasileña o sus élites, a menudo no saben claramente la importancia de la integración y, en este caso, los grandes medios de comunicación normalmente juegan un papel poco ético, exponiendo las relaciones bilaterales en el subcontinente prácticamente como actos de altruismo desinteresado, compasión y generosidad emanados desde Brasilia, que descuidaría y desampararía de la protección de los "intereses nacionales". Más específicamente, el Consejo de Defensa Suramericano no debe ser visto como un experimento exótico o una especie de Larmata Brancaleone liderada por Brasil y, en este sentido, es tangible su acción como un agente de desecuritización de temas y percepciones.

Como ha definido un especialista, "amplio en materia de propósitos y limitado en concreciones, el Consejo representa la decisión de un grupo de países integrantes de un nuevo bloque o proyecto de integración regional, UNASUR, de sentarse a hablar sobre una cuestión de interés común y sobre la cual nunca habían conversado: la defensa" (Ugarte 2011). Eso no significa que se niegue la existencia de diversidades de toda índole: desde conceptos hasta maneras de entenderse la defensa, intereses estratégicos, capacidades, tendencias políticas o vinculaciones con la superpotencia global, entre otros puntos.

A lo largo de la primera década de este siglo, período que se tiene como de ascensión del que se nombró "regionalismo post hegemónico", las reuniones no solamente aumentaron, sino que también se implementaron nuevos ajustes institucionales. Como resultado final, constatamos en este breve balance la dinámica, avances y desafíos en los campos de seguridad y defensa en el espacio geográfico suramericano que marcan el actual proceso de integración ¿o sería más adecuado calificarlo de cooperación? - en el cual destacan varias ambivalencias, inconsistencias, así como oposiciones declaradas.

Aun así, es posible afirmar que la cooperación en seguridad y defensa, aunque laudable, se va a revelar como un "gigante con pies de barro", si no se hace acompañar de una nueva cultura estratégica y de la respectiva profundización y creación de nuevas institucionalidades en los ámbitos económico, social y cultural, entre otros.

El camino es largo, tortuoso y resbaladizo, pero creemos que con el CDS se abrió una posibilidad, a pesar de los desafíos que persisten. En ese sentido, esta contribución, lejos de pretender agotar el tema en cuestión, se propone entregar una reflexión en un área aún poco explorada en Brasil y en el subcontinente, añadiéndose a otros intentos de interpretación de América del Sur contemporánea y el papel que ésta ha ejercido en lo que se refiere a la cooperación en seguridad y defensa en este siglo XXI.
La construcción de un nuevo modelo de seguridad en América del Sur: una perspectiva desde Brasil

Alexandre Fuccille 
Abdul-hak A. P. Neves (2013), O Conselho de Defesa Sul-Americano: objetivos e interesses do Brasil (CDS), FUNAG, Brasília.

Buzan B., Waever O. (2003), Regions and Powers: The Structure of International Security. Cambridge University Press, Cambridge.

Carvalho L. Arquimimo de (2009), Conselho de Defesa Sul-Americano, en: Carvalho L. Arquimimo de, Vera C. Garay, Peña y J. Cayón (orgs.), Segurança e defesa na América Latina, Juruá, Curitiba.

Colombo S., Frechero J. Ignacio (2012), Yes we can? A política externa de Obama para a América Latina: da decepção à autonomização da região, "Contexto Internacional", vol. 34 (1), pp. 189-222.

Donadio M. (2011), Visões estratégicas na América do Sul, en: Oliveira M. A. Guedes de (org.), Comparando a Defesa Sul-Americana, Editora Universitária da UFPE, Recife.

Dupas G., Oliveira M. Fernandes de (2008), A União Sul-Americana de Nações, en: Ayerbe L. F. (org.), Novas lideranças políticas e alternativas de governo na América do Sul, Editora UNESP; Programa San Tiago Dantas de Pós-Graduação em Relações Internacionais da UNESP, Unicamp e PUC-SP, São Paulo.

Fuccille A., Rezende L. Pereira (2013), Complexo Regional de Segurança da América do Sul: uma nova perspectiva, "Contexto Internacional", no 1, pp. 77-104, disponible en: http:// www.scielo.br/pdf/cint/v35n1/a03v35n1.pdf, fecha de consulta: 30 junio 2015.

Herz J. H. (1950), Idealist Internationalism and the Security Dilemma, "World Politics", vol. 2 (2), pp. 157-180, January.

Hurrel A. (1998), An emerging security community in South America?, en: Adler E., Barnett M. (ed.), Security Communities. Cambridge University Press, Cambridge.

Kacowicz A. M. (2005), The Impact of Norms in International Society: The Latin American Experience, 1881-2001, University of Notre Dame Press, Notre Dame.

Mares D. R. (2001), Violent Peace: Militarized Interstate Bargaining in Latin America, Columbia University Press, New York.

Medeiros Filho O. (2010), Conselho de Defesa Sul-Americano: Demandas e Agendas, disponible en: http://www.brasa.org/_sitemason/files/fLdvB6/Oscar\%20Medeiros\%20Filho.pdf, fecha de consulta: 13 julio 2015.

Pagliari G. de Conti (2010), O Brasil e a segurança na América do Sul, Juruá, Curitiba.

Saint-Pierre H. L., Palacios Junior A. Montoya Correa (2014), As medidas de confiança no Conselho de Defesa Sul-americano (CDS): análise dos gastos em Defesa (2009-2012), "Revista Brasileira de Política Internacional", vol. 57 (1), pp. 22-39.

Soares S. Alves (2011), Contendores apaziguados ou partícipes da cooperação? As percepções sobre ameaças e cooperação nas políticas de defesa de Argentina, Brasil e Chile na década de 1990, en: Oliveira M. A. Guedes de (org.), Comparando a Defesa Sul-Americana, Editora Universitária da UFPE, Recife.

Teixeira A. (2011), O Conselho de Defesa Sul-Americano da UNASUL, en: Oliveira M. A. Guedes de (org.), Comparando a Defesa Sul-Americana, Editora Universitária da UFPE, Recife.

Ugarte J. M. (2011), El Consejo de Defensa Suramericano: Balance y perspectivas, s.d.

Villa R. A. D., Viana M. T. (2010), Security issues during Lula's administration: from the reactive to the assertive approach, "Revista Brasileira de Política Internacional", vol. 53 (special edition), pp. 91-114. 